\title{
Does IO affect the functional brain network involved in pseudoword reading in students with reading disability? A magnetoencephalography study
}

\author{
Panagiotis G. Simos ${ }^{1}$, Roozbeh Rezaie ${ }^{2,3}$, Andrew C. Papanicolaou ${ }^{2,3}$ and Jack M. Fletcher ${ }^{4}$ \\ 1 Department of Psychiatry, School of Medicine, University of Crete, Herakleion, Crete, Greece \\ ${ }^{2}$ Department of Pediatrics, Division of Clinical Neurosciences, College of Medicine, University of Tennessee Health Science Center, Memphis, TN, USA \\ ${ }^{3}$ Neuroscience Institute-Le Bonheur Children's Hospital, Memphis, TN, USA \\ ${ }^{4}$ Department of Psychology, University of Houston, Houston, TX, USA
}

\section{Edited by:}

Vasileios Kokkinos, St. Luke's

Hospital, Greece

Reviewed by:

Fumiko Hoeft, University of California

San Francisco, USA

John D. E. Gabrieli, Massachusetts

Institute of Technology, USA

\section{${ }^{*}$ Correspondence:}

Panagiotis G. Simos, Department of

Psychiatry, School of Medicine,

University of Crete, Herakleion,

Crete 71003, Greece

e-mail:akis.simos@gmail.com
The study examined whether individual differences in performance and verbal IQ affect the profiles of reading-related regional brain activation in 127 students experiencing reading difficulties and typical readers. Using magnetoencephalography in a pseudoword readaloud task, we compared brain activation profiles of students experiencing word-level reading difficulties who did $(n=29)$ or did not $(n=36)$ meet the IQ-reading achievement discrepancy criterion. Typical readers assigned to a lower-IQ $(n=18)$ or a higher $I Q$ $(n=44)$ subgroup served as controls. Minimum norm estimates of regional cortical activity revealed that the degree of hypoactivation in the left superior temporal and supramarginal gyri in both RD subgroups was not affected by $\mathrm{IQ}$. Moreover, IQ did not moderate the positive association between degree of activation in the left fusiform gyrus and phonological decoding ability. We did find, however, that the hypoactivation of the left pars opercularis in $\mathrm{RD}$ was restricted to lower-IQ participants. In accordance with previous morphometric and fMRI studies, degree of activity in inferior frontal, and inferior parietal regions correlated with $\mathrm{IO}$ across reading ability subgroups. Results are consistent with current views questioning the relevance of IQ-discrepancy criteria in the diagnosis of dyslexia.

Keywords: magnetoencephalography, functional brain imaging, reading, dyslexia, intelligence

\section{INTRODUCTION}

An enduring issue concerning both the definition and diagnosis of developmental reading disability $(\mathrm{RD})$ rests in the use of measures of general cognitive ability (operationalized as IQ scores). Traditional definitions of $\mathrm{RD}$ not only require general intellectual functioning within the normal range but often set a minimum degree of discrepancy between reading achievement standard scores and IQ in order for a diagnosis of $\mathrm{RD}$ to be made (Fletcher et al., 2007). In earlier studies, poor readers who read at levels consistent with their IQ scores (low achievers) differed from poor readers who read well below their IQ levels (IQ-discrepant) on a variety of attributes, including prognosis, severity of reading difficulties, gender, and some cognitive abilities (Rutter and Yule, 1975). Subsequent research, however, has not replicated these findings, suggesting that these earlier findings reflected inclusion of children with intellectual disabilities and brain injury. More recent meta-analyses have reported little difference between IQ-discrepant and low achieving poor readers on reading skills and cognitive abilities closely related to reading (phonological awareness, rapid naming, working memory, and vocabulary; Hoskyn and Swanson, 2000; Stuebing etal., 2002; see also Fletcher etal., 1994; Stanovich and Siegel, 1994). Further evidence suggested that the two subgroups of RD children show comparable response to intervention and overall prognosis (Francis etal., 1996; Vellutino etal., 2000; Stuebing etal., 2009).
Despite these findings, it is common in neuroimaging studies to select participants based on either IQ-discrepancy criteria or to use relatively high IQ cut-offs. Such an approach is supported by evidence for small differences in the heritability of reading skills in poor readers with higher and lower IQ scores (Wadsworth et al., 2010). In this line of research, assessments of large twin samples have suggested that RD in children with higher IQ scores has a stronger genetic etiology. These practices and findings would suggest differences in the functional organization of the brain networks engaged during reading tasks between IQ-discrepant and low achieving poor readers.

Numerous neuroimaging studies using fMRI and magnetoencephalography have documented reduced activation among students with $\mathrm{RD}$, as compared to typical readers. These differences are most apparent in left hemisphere regions purportedly involved in phonological processes, namely in the posterior portion of the superior temporal gyrus, and the supramarginal gyrus (Temple et al., 2001; Shaywitz et al., 2002; Simos et al., 2007, 2011), often extending into the adjacent angular gyrus (Shaywitz etal., 1998; Temple etal., 2001; Simos et al., 2007). Several studies have also reported reduced activation in left hemisphere ventral and/or lateral occipitotemporal regions believed to be involved in orthographic/graphemic processing (Shaywitz et al., 2002; McCrory et al., 2005; Cao et al., 2006; Hoeft et al., 2007a; van der Mark etal., 2009). Reports of changes in inferior frontal activity during reading in $\mathrm{RD}$ are more 
variable (e.g., Shaywitz et al., 2002; Cao et al., 2006; Hoeft et al., 2007a).

The research question addressed in the current study is whether variability in general cognitive ability as indexed by performance and verbal IQ makes a difference in neuroimaging studies as it seems to make in behavioral genetic studies. To our knowledge there has been a single attempt to assess the neurophysiological plausibility of the IQ-discrepancy criterion: Tanaka et al. (2011) reported fMRI data obtained during performance of a wordrhyme task from 69 struggling readers (identified by a score below the 25th percentile on a word reading accuracy test) and 62 typical readers. The RD sample was further subdivided into a highand a low-IQ group as defined by a cutoff standard score of 90 points on the Peabody Picture Vocabulary Test. This study did not explicitly manipulate the IQ-discrepancy criterion. However, although both groups of RD students scored, on average, lower on this measure of verbal IQ than the group of typical students, the majority of students in the high-IQ group met the IQ-discrepancy criterion, whereas the majority of students in the low-IQ group did not. Analyses focusing on three ROIs per hemisphere revealed that both RD groups showed reduced hemodynamic activation in the left ventral occipitotemporal region (fusiform gyrus) and the left inferior parietal lobule (mainly the supramarginal gyrus). Complementary whole brain analyses showed that none of the activation sites that distinguished typical from poor readers differed in the degree of hemodynamic activity between the two groups of poor readers.

The results of the large-scale study by Tanaka etal. (2011) are important in demonstrating that the functional organization of the brain network involved in phonological processing of print does not vary as a function of verbal IQ in poor readers. These findings leave, however, the possibility open that poor readers may engage additional regions depending on their general cognitive ability. Although regions such as the dorsolateral prefrontal cortex, the superior parietal lobule, and the anterior cingulate are not generally considered parts of the brain network specialized for reading, there is increasing evidence implicating these regions in IQ-related functions, including spatial cognition, executive functions, and attention. For instance, the degree of task-related hemodynamic activity in the dorsolateral prefrontal cortex is affected by IQ (Perfetti et al., 2009), whereas the degree of age-related increase in cortical thickness in superior frontal cortices is significantly higher among high-IQ persons over the age range covered by the present study (Shaw et al., 2006). Concurrent recruitment of dorsolateral prefrontal and posterior superior parietal cortices has been linked to the ability to solve non-verbal reasoning problems similar to those included in the majority of common IQ tests (Kroger et al., 2002; Choi et al., 2008). Further, the importance of posterior superior parietal, along with superior, middle, and inferior prefrontal, regions for general intelligence and executive function is supported by recent voxel-based lesion studies (Gläscher et al., 2010; Barbey et al., 2012). The anterior cingulate gyrus has been implicated in attention especially under increasing task difficulty conditions-as when reading rapidly presented, unfamiliar printed stimuli by RD students (Cattinelli et al., 2013). An additional visual area (cuneus) was also examined in view of its suggested role in the control of visual attention (Vanni etal., 2001; Simpson etal., 2011) in tasks that include reading (Osipowicz et al., 2011).

The primary goal of the present study was to extend these fMRI findings in three directions. First, we directly compared two groups of RD students who either met or did not meet the IQ-discrepancy criterion on non-verbal IQ, as well as verbal IQ. Second, we assessed the potential moderating role of IQ in determining reading-related activity, not only among struggling readers but also among typical readers. Third, we expanded the research question from hemodynamic to neurophysiological measures occurring in real time, using magnetoencephalography. We compared brain activation profiles of students experiencing word-level reading difficulties $(n=65)$ who either met the IQ-reading achievement discrepancy criterion $(n=29)$ or did not meet this criterion $(n=36)$ while performing a pseudoword read-aloud task. Two groups of typical readers served as comparisons: students in the lower-IQ group served as controls for the non-discrepant RD subgroup $(n=18)$, whereas students in the higher-IQ group $(n=44)$ served as controls for the IQ-discrepant RD subgroup. We hypothesized that the children with higher IQ scores, whether verbal or performance, would show increased activation in regions outside the reading network related to language and spatial cognition, but not in reading-related areas.

\section{MATERIALS AND METHODS PARTICIPANTS}

Magnetoencephalography data were obtained from 127 students aged 6.5-14.3 years. The sample included 65 children experiencing reading difficulties ( $\mathrm{RD}$ group), as indicated by scores below the 25th percentile (standard score of 90) on the Basic Reading composite [average of Word Attack (WA) and Letter-Word Identification (LWID)] subtest scores of the Woodcock-Johnson Tests of Achievement-III [W-J III], Woodcock et al., 2001). A group of 62 children who had never experienced reading difficulties and had scores $>92$ on the basic reading composite index served as controls. Table 1 displays demographic and psychoeducational information for each of the four groups of participants, which were comparable on age, ethnicity, handedness, and Performance IQ (PIQ). All participants had full scale (FSIQ) scores $>77$ on the Wechsler Abbreviated Scale of Intelligence (Wechsler, 1999) and no history or current indications of ADHD as indicated by $T$ scores $<55$ on the attention problems scale of the parent form of the Child Behavior Checklist (CBCL; Achenbach, 1991) or a mean score lower than 1.67 on the inattention and hyperactivity-impulsivity scales of the parent-completed Swanson, Nolan, Achenbach, Pelham questionnaire (SNAP-IV; Swanson, 1992) indicating low risk for ADHD (Chen etal., 1994). Fourteen low-achieving and nine typically achieving students were excluded from the current cohort for not meeting the aforementioned ADHD criteria.

Following Tanaka et al. (2011) and Wadsworth et al. (2010), each group was further divided into two subgroups based on FSIQ. $\mathrm{RD}$ students in the higher-IQ, discrepant group scored at least one standard deviation higher on FSIQ than on the WJ Reading Composite (range: $96-123$ points). In all cases, students in the lower-IQ, non-discrepant group had FSIQ scores within one 
Table 1 | Demographic data and performance on standardized tests for each group of participants (mean and SD in parentheses).

\begin{tabular}{|c|c|c|c|c|}
\hline & $\begin{array}{l}\text { IQ-discrepant } \\
\text { (higher IQ; } n=29 \text { ) }\end{array}$ & $\begin{array}{l}\text { Non-discrepant } \\
\text { (lower IQ; } n=36 \text { ) }\end{array}$ & Lower IQ $(n=18)$ & Higher IQ $(n=44)$ \\
\hline Gender (m/f) & $22 / 7$ & $25 / 11$ & $13 / 5$ & $26 / 18$ \\
\hline Age (mo) & $135.41(20.52)$ & $140.54(26.23)$ & 107.3 (33.3) & 116.33 (26.19) \\
\hline LWID & $74.45(8.16)$ & 75.33 (11.97) & $108.38(11.01)$ & 107.48 (11.97) \\
\hline Spelling & $73.68(16.56)$ & $75.52(11.19)$ & $113.62(13.41)$ & 110.93 (12.65) \\
\hline VIO & $109.48(12.31)$ & $90.37(8.21)$ & 89.06 (12.83) & $113.42(14.90)$ \\
\hline $\mathrm{PIO}$ & 105.09 (11.38) & $87.03(12.01)$ & $92.44(10.60)$ & $106.89(14.26)$ \\
\hline$\%$ correct & 51.37 (17.84) & 56.67 (16.58) & $93.09(2.87)$ & 88.51 (11.32) \\
\hline
\end{tabular}

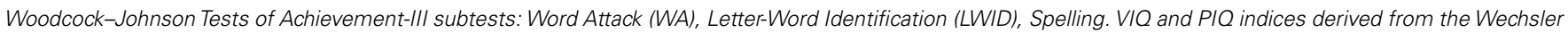
Abbreviated Scale of Intelligence. \% correct: Percent correct pseudowords read aloud on the activation task in the scanner.

SD from their reading achievement standard scores (range: 77-96 points). A median split (corresponding to an FSIQ score of 95) was set to assign typical readers into a higher- (range: 95-131) and a lower-IQ group (range: 77-94 points).

The two RD groups did not differ on age, on any of the standardized reading measures or on in-scanner performance on the activation task $(p>0.19)$. By definition the IQ-discrepant RD group had higher average VIQ and PIQ than the non-discrepant RD group, $F(1,64)=56.11, p=0.0001$ and $F(1,64)=19.68$, $p=0.0001$, respectively. The two groups of typical readers were also comparable on age, in-scanner performance and standardized reading measures $(p>0.09)$. By definition, the Higher-IQ subgroup of typical readers had higher VIQ and PIQ score than the Lower IQ subgroup of typical readers, $F(1,61)=25.82, p=0.0001$ and $F(1,61)=10.54, p=0.001$, respectively.

Across IQ subgroups, typical readers were older than their $\mathrm{RD}$ peers [main effect of RD Group: $F(1,126)=34.22$, $p=0.0001]$, and scored higher (main effects of RD Group) on WA, $F(1,126)=439.12, p=0.0001$, LWID, $F(1,126)=239.71$, $p=0.0001$, Spelling, $F(1,126)=254.09, p=0.0001$, and inscanner performance: $F(1,126)=87.32, p=0.0001$. By design the IQ-discrepant RD subgroup and the High-IQ typical reading group were comparable on PIQ and VIQ $(p>0.07)$, as were the non-discrepant RD subgroup and the Lower IQ subgroup of typical readers $(p>0.1)$.

\section{PROCEDURE}

\section{Task}

Each participant was tested on a pseudoword read-aloud task involving three-letter pronounceable non-words (e.g., lan), subtending $2.0^{\circ}$ of visual angle. For each task 100 stimuli were presented randomly arranged in four blocks of 25 items each. Stimuli were presented for $1 \mathrm{~s}$, one at a time (with a randomly varied interstimulus interval of 3-4 s), through a Sony LCD projector (Model VPL-PX21) on a back-projection screen located approximately $60 \mathrm{~cm}$ in front of the participant. Participants were instructed to name read aloud each letter string immediately after it had disappeared from the screen. Prior to each scan children were asked to practice this response strategy while magnetic activity was monitored online to ensure that movement artifacts associated with articulation systematically occurred only after the end of the recording epoch. Epochs containing such movement artifacts (when, occasionally, verbal responses were produced earlier than instructed) were not included in the data analyses.

\section{Imaging procedures}

Magnetoencephalography recordings were obtained with a wholehead neuromagnetometer array (4-D Neuroimaging, Magnes WH3600), that consisted of 248 first-order axial gradiometer coils, housed in a magnetically shielded chamber and arranged to cover the entire head surface. The magnetic flux measurements were digitized at $250 \mathrm{~Hz}$, filtered with a bandpass filter between 0.1 and $20 \mathrm{~Hz}$ and subjected to baseline adjustment (using the $150 \mathrm{~ms}$ prestimulus recording) and to a noise reduction algorithm that is part of the $4 \mathrm{D}$-Neuroimaging software. The single-trial event-related field segments (ERFs) in response to 60-80 stimulus presentations, were averaged after excluding those containing eye movement or other myogenic or mechanical artifacts.

To identify the intracranial origin of ERFs, the magnetic flux distribution recorded simultaneously over the entire head surface at successive points (4 ms apart) was analyzed using a minimum norm model to obtain estimates of the time-varying strength of intracranial currents (Hamalainen M. MNE Software Users Guide. Version 2.5. Charlestown, MA: 2006). This method affords greater spatial resolution and allows detection of simultaneous magnetic sources distributed along the entire cortical surface. The model assumes a continuous distribution of current along the cortical surface which has some minimum norm (Hämäläinen and Ilmoniemi, 1994). Estimated current sources were anatomically constrained by an MRI-derived surface model of each participant's brain (T1-weighted: TR $13.6 \mathrm{~ms}$; TE $4.8 \mathrm{~ms}$; recording matrix $256 \times 256$ pixels, 1 excitation, $240 \mathrm{~mm}$ field of view, and $1.4 \mathrm{~mm}$ slice thickness), obtained on a Philips $3 \mathrm{~T}$ scanner with SENSE (Sensitivity Encoding) technology. 
This surface model was generated by a fully automated cortical surface reconstruction procedure using FreeSurfer software (Dale et al., 1999) for producing a detailed geometric description (regular tessellation of the cortical surface consisting of equilateral triangles known as vertices) of the gray-white matter boundary of the neocortical mantle and the mesial temporal lobe. Each hemisphere consisted of approximately 150,000 vertices (depending on each subject's cortical surface area). For estimating current sources, the MNE software requires the Freesurfer-derived cortical surface reconstruction for defining the boundaries of a solution source space. A grid-spacing of $7 \mathrm{~mm}$ was used to construct icosahedrons to decimate the number of vertices from 150,000 to approximately 3,000 per hemisphere. Additionally, the MNE software was used to construct a single compartment boundary element model using triangular tessellations to model each vertex as a potential current dipole perpendicular to the cortical surface during the forward calculations. The inverse solution was subsequently reduced to obtain an estimate of the scalar distribution of dipole strength across current sources within orientation-specific cortical patches of vertices (Dale et al., 1999). Co-registration of each MEG dataset with its corresponding MRI dataset was performed using an automated co-registration routine within MNE which aligns digitization points in the MEG headshape file with the fiducial points demarcated on the outer skin surface reconstruction of the MRI.

As in our previous report from this cohort (Simos et al., 2011) activity was examined in the following set of ROIs (separately in each hemisphere): superior temporal gyrus (STG; BA 22); supramarginal gyrus (SMG; BA 40); angular gyrus in the IPL (BA 39); pars opercularis and triangularis of the inferior frontal gyrus (BA 44/45); fusiform gyrus (BA 37); and lateral occipito-temporal cortex (BA 19). Additional areas hypothesized to be involved in spatial cognition, attention, and executive functions that may lead to higher IQ scores included the superior parietal lobule (BA 7), rostral middle (BA 46/9), and superior frontal cortices (BA 8/9); anterior cingulate (BA 24), and cuneus (BA 19). The program outputs a current estimate value for each voxel and each $4 \mathrm{~ms}$ time point. This value was then used to compute the dependent measure used in subsequent analyses namely the average current across all voxels defining each of the ROIs listed above and across all of the $4 \mathrm{~ms}$ time points comprising 13 successive $50 \mathrm{~ms}$ time bins (100-150, 150-200 ms, etc., up to $800 \mathrm{~ms}$ ).

\section{ANALYSES}

The overall model used in the group-level analyses was in the form of a RD group (RD vs. typical) by IQ group (higher vs. lower) ANCOVA with age as a covariate. Significant interactions were followed by one-way, simple main effects tests. The dependent measure in each analysis was averaged degree of activity for each ROI and 50-ms time window starting at $100 \mathrm{~ms}$ post-stimulus onset and ending at $750 \mathrm{~ms}$. A nominal alpha level of 0.0038 was used to correct for family wise type I error rate.

Associations between degree of activity and achievement/IQ measures were then explored for those ROIs and time windows where significant main effects or interactions were found in the ANCOVAs. In addition, correlations between degree of activity in BA 37/19 with reading achievement and IQ were conducted as planned analyses, given that in our previous report (Simos et al., 2011) significant associations were found with reading achievement despite lack of significant differences between RD Groups in these regions. For ROIs and time windows where significant correlations with reading achievement scores were found, we tested the regulating role of IQ through moderated regression models conducted with SPSS macros developed by Hayes (model 2; Hayes, 2013). In this model the following equation was used to estimate reading achievement scores:

$$
Y=\mathrm{i}_{\mathrm{y}}+\mathrm{c}^{\prime} X+\mathrm{b}_{1} M+\mathrm{b}_{2} W+\mathrm{b}_{3} X * M+\mathrm{b}_{4} X * W+\mathrm{e}_{\mathrm{y}},
$$

where $Y$ represents WJ-WA or WJ-Letter Word Identification subtest standard scores, $X$ represents degree of activity in a particular ROI and time bin, and $M$ and $W$ indicate the moderator variables (VIQ and PIQ, respectively). All variables were centered to their respective grand means before entered into the analyses.

\section{RESULTS}

\section{GROUP-LEVEL ANALYSES}

ANCOVA results revealed significant interactions of RD Group and IQ Group for left pars opercularis activity at 500-550 $[F(1,122)=9.11, p<0.003]$ and at $550-600 \mathrm{~ms}$ post-stimulus onset $[F(1,122)=11.22, p<0.001$; see Figure 1]. Follow up tests showed that RD students who met the IQ-discrepancy criterion had higher degree of activity than Lower-IQ RD students at both time windows $[F(1,62)=8.64, p<0.003$ and $F(1,62)=10.99$, $p<0.002$, respectively]. Moreover, lower degree of activity in this region was found for poor as compared to typical readers of overall lower, yet comparable IQ [Lower-IQ RD students vs. Lower-IQ typical readers: $F(1,51)=11.21, p<0.002$ and $F(1,51)=15.04$, $p<0.0001$, respectively]. Higher-IQ RD and Higher-IQ typical readers demonstrated comparable degree of activity $(p>0.3)$. There were no other significant two-way interactions.

There were, however, significant main effects of IQ Group in three regions, indicating higher degree of activity for Higher-IQ than for Lower-IQ students: the left angular gyrus [between $450-500, F(1,122)=8.52, p<0.003$, and $600-650 \mathrm{~ms}$, $F(1,122)=8.22, p<0.0037$, the right angular gyrus [between 600 and $700 \mathrm{~ms}, F(1,122)=8.84, p<0.003$ and $F(1,122)=9.30$, $p<0.003$ ] and the left cuneus [between 300 and $450 \mathrm{~ms}$, $F(1,122)=10.97, p<0.001, F(1,122)=8.44, p<0.003$, and $F(1,122)=8.74, p<0.003]$.

Finally, as expected based on our previous results (Simos et al., 2011) average activity in the left STG [between 350-400, $F(1,122)=11.25, p<0.001$ and $450-500 \mathrm{~ms}, F(1,122)=11.83$, $p<0.001$ ] and left SMG [between 400 and $450 \mathrm{~ms}$, $F(1,122)=8.15, p<0.003$ ] was stronger among typical than RD students, independent of IQ, as indicated by main effects of RD Group.

\section{BRAIN-ACHIEVEMENT/IO ASSOCIATIONS}

Correlational analyses conducted on the entire sample $(n=127)$ indicated that degree of activity in the left pars opercularis was positively associated with VIQ $(r=0.21$ at $400-450 \mathrm{~ms}$, controlling for PIQ and reading achievement) but not with PIQ or reading achievement $(r<0.1)$. Conversely, degree of activity in the left 


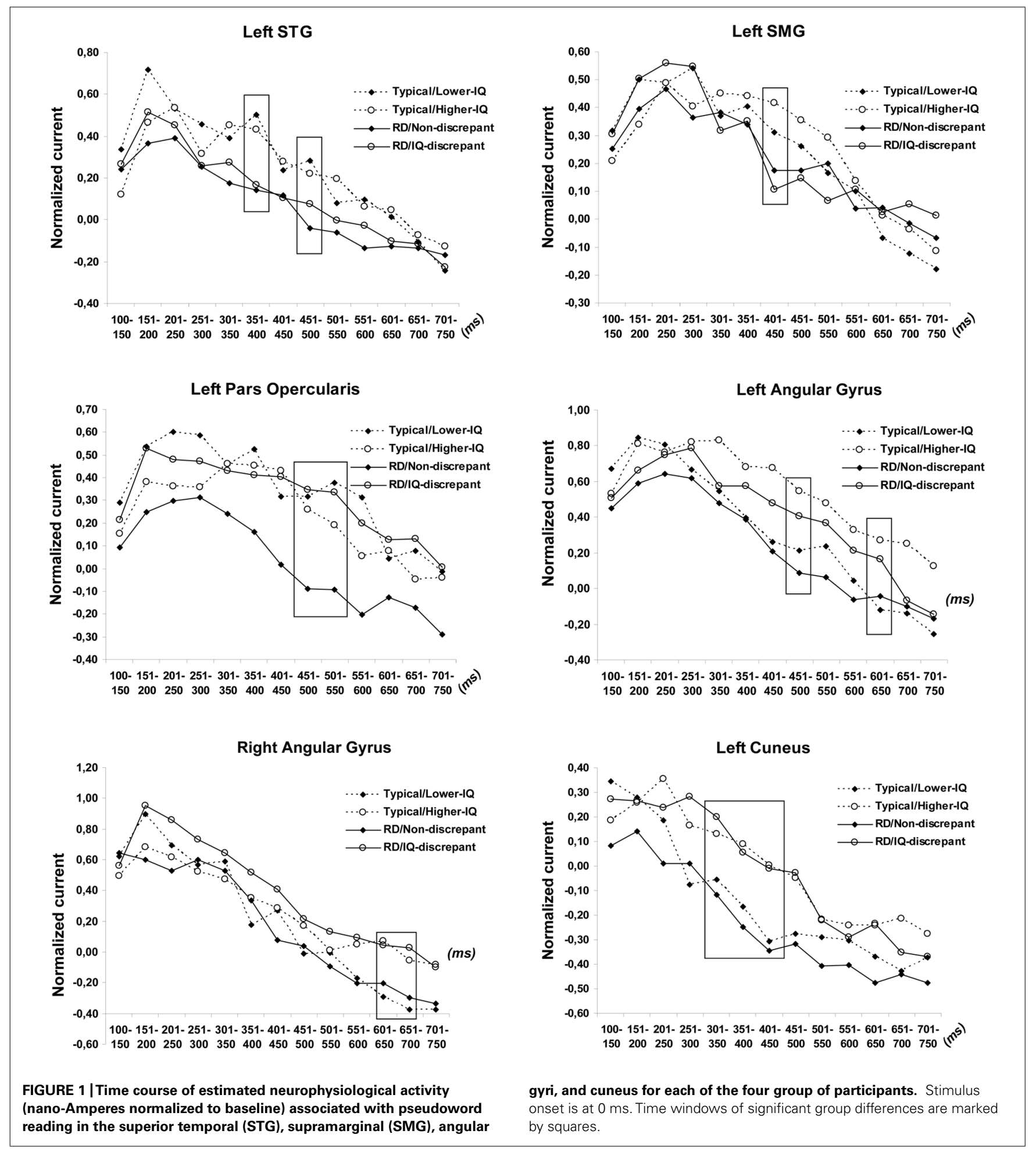

angular gyrus ( $r=0.24$ at $450-500 \mathrm{~ms})$ and cuneus $(r=0.30$ at $300-350 \mathrm{~ms}$ and $r=0.28$ at $450-500 \mathrm{~ms}$ ) was positively associated with PIQ (controlling for VIQ and reading achievement) but not with VIQ or reading achievement $(r<0.2)$. These correlations account for small amounts of the association of IQ and brain function.

WJ-WA scores were positively associated with left STG activity ( $r=0.31$ at $350-400 \mathrm{~ms})$ as reported previously in a partially overlapping sample (Simos et al., 2011). Although activity in the left fusiform gyrus did not vary as a function of RD- or IQ-group, planned correlational analyses replicated the significant positive association between left fusiform activity at 200-250 ms and 
performance on the WJ-WA task ( $r=0.37, p=0.003)$, which was restricted to typical readers $(r=-0.08$ in the entire RD group). Correlations between left fusiform activity and IQ scores was $<0.15$ across all time windows.

\section{MODERATED REGRESSION ANALYSES}

A stronger test of the role of IQ is the moderated regression model of the association between (a) left STG activity at 350-400 ms and (b) left fusiform activity at 200-250 ms and WJ-WA scores in the entire sample, including VIQ and PIQ measures as moderators. This analysis did not show that different IQ scores served as significant moderating effects $(p>0.2)$.

\section{DISCUSSION}

The present study assessed whether reading skill-related variability in the degree of regional cortical activity varied as a function of participant verbal or PIQ. Our findings largely replicated the earlier work of Tanaka et al. (2011) using assessments of IQ that included measures of fluid and crystallized intelligence and a composite that represented a more traditional index than a receptive vocabulary measure. In addition, our higher-IQ RD subgroup met a formal IQ-discrepancy standard. As hypothesized, the most consistently found signs of aberrant brain function in $\mathrm{RD}$, involving the left temporo-parietal cortex (e.g., Pugh et al., 2000; Joseph et al., 2001; Jobard et al., 2003) were not moderated by IQ. There was one exception to this trend involving the left pars opercularis, where hypoactivation in $\mathrm{RD}$ vs. typical readers depended upon IQ: this effect was observed only among lower-IQ participants. Across participants, degree of activity in this region was positively related to VIQ but did not correlate with reading skill. This finding may help account for discrepancies across previous neuroimaging studies with respect to reading skill-related effects on the degree of left inferior frontal gyrus activation. Some studies have reported increased activation among RD students and attributed this finding to compensatory processes (Shaywitz et al., 2002; Cao et al., 2006), whereas others failed to find group differences (Temple et al., 2001; Maisog et al., 2008; Simos et al., 2011). Increased activation depending on the type of comparison has been reported in another study (RD > age-matched controls whereas no difference was noted between RD and reading-matched controls; Hoeft et al., 2007a). There have also been reports of group differences in the opposite direction (Richards et al., 2002; Simos et al., 2007). The present results highlight a potential confounding role of VIQ in these earlier reports which is discussed further below in the context of the two main topics addressed by the present study, namely associations between brain activation, individual reading ability, and IQ.

\section{FUNCTIONAL BRAIN CORRELATES OF GENERAL COGNITIVE ABILITY}

We identified three left hemisphere sites where degree of activity varied with general cognitive ability: the angular gyrus, pars opercularis, and cuneus. Correlational analyses suggested that activity in the angular gyrus and cuneus was more closely linked to PIQ, whereas a positive association was found between pars opercularis activation and VIQ. Although small but significant, these correlations are not surprising given the rising body of neuroimaging evidence regarding associations of morphometric and/or hemodynamic measures with IQ in children and adolescents. These include correlations between fractional anisotropy (Tamnes et al., 2010), cortical thickness (Kamara et al., 2009), gray matter volume (Taki et al., 2012), and local efficiency indices (derived from resting-state fMRI data; $\mathrm{Wu}$ et al., 2013) in inferior parietal and/or inferior frontal cortices with IQ.

Determining the nature of brain-IQ associations is rendered exceedingly difficult, in view of the multitude of available, complementary anatomical, and physiological measures of brain integrity and of the multidimensional nature of IQ tests and component cognitive processes each IQ task involves. A promising approach, that is currently explored by our group, involves first computing measures of regional interdependence between MEG-derived cortical activation time series, serving to establish real-time functional connectivity profiles during task performance. Specific elements of these profiles, along with more traditional measures of the degree of regional brain activity, may then be linked to particular reading-related processes (such as phonological decoding or word recognition). Anatomic features of the purported functional network (e.g., cortical thickness of implicated regions and anisotropy of underlying white matter) may then be used to refine specific network elements (see for example Hoeft et al., 2007b).

A further issue that deserves consideration is the potential confounding effect of age in the associations between IQ, reading achievement and brain activity. This possibility is raised by evidence that heritability of IQ varies with age (Brant et al., 2013), as does the anatomical distribution of associations between IQ and cortical thickness (Shaw et al., 2006).

\section{CORTICAL REGIONS INVOLVED IN PHONOLOGICAL DECODING}

Two sites were identified for showing neurophysiological activity that varied as a function of phonological decoding ability, as measured outside the scanner through standardized tests: the superior temporal and fusiform gyri in the left hemisphere. Further, at the group level, RD students showed reduced degree of activity in the left supramarginal gyrus compared to typical readers. Similar findings have been reported by several studies (Cao et al., 2006; Hoeft et al., 2007a; Maisog et al., 2008; van der Mark et al., 2009; Simos et al., 2011; Tanaka etal., 2011), and is generally interpreted as highlighting the key role of cortex in the temporo-parietal junction for sub-word level phonological processing and analysis (Beauvois and Derouesne, 1979; Caplan et al., 1995; Scott et al., 2000; Specht et al., 2003; Jacquemot et al., 2003; Majerus et al., 2005).

Another region that is often implicated in reading and $\mathrm{RD}$ is the angular gyrus (in the IPL). In the present study, we found associations between IQ and activity in this region, independently of individual reading ability which, however, do not preclude its regular involvement in the reading process, given that these were observed during performance of a reading task. Moreover, a role of the IPL within the brain network for reading has been established by lesion (Philipose et al., 2007), electrocortical stimulation (Roux et al., 2004), and imaging studies (Booth et al., 2003). Nevertheless, reports implicating the left IPL in the pathophysiology of RD are less consistent across studies (see for instance Eden and Zeffiro, 
1998; Pugh et al., 2000; Temple et al., 2001; Simos et al., 2007). In addition, this area of the brain is involved in a wide range of linguistic and non-linguistic cognitive processing reflecting its role as a cross-modal association area.

With respect to activity in the fusiform gyrus, the positive association between degree of activation and individual differences in phonological decoding ability is in accordance with previous neuromagnetic (Simos et al., 2011) and hemodynamic studies (Shaywitz etal., 2003). It is widely postulated that the role of ventral occipitotemporal cortices in phonological decoding tasks (and reading in general) concerns processing and storage of orthographic information (graphemic patterns; Tarkiainen et al., 1999; McCandliss et al., 2003; Philipose et al., 2007).

Reading disability group differences in left inferior frontal activation have been quite variable in both degree and direction with some studies reporting increased activation in RD (Shaywitz et al., 2002; Cao et al., 2006) and others reduced activation (Richards et al., 2002; Simos et al., 2007). Such inconsistencies and our failure to establish a significant (across or within RD groups) association between degree of left pars opercularis activity and decoding skill raise questions regarding the role of this region within the brain network responsible for phonological decoding. Our finding that only lower-IQ RD students demonstrated decreased pars opercularis activity as compared to typical readers of comparable IQ, may suggest that deficient engagement of this region for decoding in struggling readers depends upon cognitive ability and especially general language ability. It has been proposed that increased activity in this region in RD indicates increased "neural effort" in order to cope with the higher level of difficulty imposed by the decoding task (compared to the level of difficulty experienced by typically achieving readers; e.g., Hoeft et al., 2007a). Current results suggest a link between pars opercularis activation and verbal functions (as indicated by a significant correlation with VIQ in the entire sample). Failure to demonstrate this compensatory neural response by lower-IQ RD students may, therefore, indicate reduced capacity to engage in compensatory strategies for decoding. Such strategies may include articulatory recoding and/or access to stored real-word phonological representations. Moreover, recruitment of such compensatory strategies would pose greater demands upon working memory, as compared to the application of a straightforward, well-learned phonological conversion routine. Thus, lack of inferior frontal hyperactivity is also consistent with the key role of working memory in cognitive control and IQ (Conway et al., 2003; Wiley et al., 2011).

In sum, the present results largely confirm and extend the earlier report of Tanaka etal. (2011). A novel finding that may have implications for future imaging studies of RD concerns the potential confounding role of VIQ in assessing reading achievement group differences on inferior frontal activation. However, the fact that this relation was with VIQ and not PIQ is consistent with other findings suggesting that lower IQ children with RD are more impaired in overall language ability (Morris et al., 1998). Additionally, positive correlations between degree of activity and IQ were found in three cortical regions - the angular gyrus and cuneus (with PIQ) and the pars opercularis (with VIQ) - in the context of a task that did not require, at least in principle, complex cognitive operations such as those tapped by mainstream IQ tests. Given that degree of activity in these regions did not correlate with reading achievement, we may surmise that they play an auxiliary role in decoding. Moreover, the temporal resolution of magnetoencephalography made it possible to determine the time windows during which neurophysiological activity correlated with reading achievement or IQ. In all cases these effects reflected activity taking place later than 200-250 ms after stimulus onset (i.e., the time window when the degree of activity in the left fusiform gyrus correlated with reading achievement). Although circumstantial, this evidence is consistent with the notion that significant activations in the angular gyrus, cuneus, and IPL were involved in a post-orthographic processing stage.

To conclude, there is not strong evidence of a need to use an IQ-discrepancy criterion in neuroimaging studies. Although there were some differences between higher and lower IQ groups in the inferior frontal and angular gyrus regions, these seem to be related to overall language proficiency and the difference in VIQ - a selection criterion - and not to reading, where the IQ groups did not differ. In addition, so long as the lower threshold is set at a level not associated with intellectual disabilities (typically above the second percentile), the range of IQ scores in the final sample does not seem critical. In fact, because the population of all children with RD has lower IQ scores than typically achieving children, matching on IQ or setting the lower threshold too close to average may result in an unrepresentative sample. In behavioral studies, there is little evidence that IQ is strongly related to prognosis, intervention response, or cognitive skills related to reading (Fletcher et al., 2007). As in the present study, there are differences in cognitive skills not related to reading. Moreover, the IQ-achievement discrepancy is no longer required in the US Individuals with Disabilities in Education Act and was abandoned by DSM-5. The reductions in VIQ associated with $\mathrm{RD}$ are just as likely caused by language weaknesses also associated with $\mathrm{RD}$ or result from $\mathrm{RD}$ and the impact of poor reading on vocabulary and other forms of language learning. Focusing on relations of RD with more discrete skills than those typically measured by IQ tests would seem an important direction for neuroimaging research. In addition, further exploration attempting to differentiate the role of the inferior frontal and angular gyrus regions in language vs. reading skills would be indicated.

\section{ACKNOWLEDGMENTS}

This research was supported in part by grant P50 HD052117 from the Eunice Kennedy Shriver National Institute of Child Health and Human Development (NICHD). The content is solely the responsibility of the authors and does not necessarily represent the official views of the NICHD or the National Institutes of Health.

\section{REFERENCES}

Achenbach, T. M. (1991). Manual for the Child Behavior Checklist/4-18 and 1991 Profile. Burlington, VT: University of Vermont.

Barbey, A. K., Colom, R., Solomon, J., Krueger, F., Forbes, C., and Grafman, J. (2012). An integrative architecture for general intelligence and executive function revealed by lesion mapping. Brain 135, 1154-1164. doi: 10.1093/brain/aws021

Beauvois, M. F., and Derouesne, J. (1979). Phonological alexia: three dissociations. J. Neurol. Neurosurg. Psychiatry 42, 1115-1124. doi: 10.1136/jnnp.42.12.1115 
Booth, J. R., Burman, D. D., Meyer, J. R., Gitelman, D. R., Parrish, T. B., and Mesulam, M. M. (2003). Relation between brain activation and lexical performance. Hum. Brain Mapp. 19, 155-169. doi: 10.1002/hbm.10111

Brant, A. M., Munakata, Y., Boomsma, D. I., DeFries, J. C., Haworth, C. M. A. Keller, M. C., et al. (2013). The nature and nurture of high IQ: an extended sensitive period for intellectual development. Psychol. Sci. 24, 1487-1495. doi: 10.1177/0956797612473119

Cao, F., Bitan, T., Chou, T.-L., Burman, D. D., and Booth, J. R. (2006). Deficient orthographic and phonological representations in children with dyslexia revealed by brain activation patterns. J. Child Psychol. Psychiatry 47, 1041-1050. doi: 10.1111/j.1469-7610.2006.01684.x

Caplan, D., Gow, D., and Makris, N. (1995). Analysis of lesions by MRI in stroke patients with acoustic-phonetic processing deficits. Neurology 45, 293-298. doi: 10.1212/WNL.45.2.293

Cattinelli, I., Borghese, N. A., Gallucci, M., and Paulesu, E. (2013). Reading the reading brain: a new meta-analysis of functional imaging data on reading. J. Neurolinguist. 26, 214-238. doi: 10.1016/j.jneuroling.2012. 08.001

Chen, W. J., Faraone, S. V., Biederman, J., and Tsuang, M. T. (1994). Diagnostic accuracy of the child behavior checklist scales for attention-deficit hyperactivity disorder: a receiver-operating characteristic analysis. J. Consult. Clin. Psychol. 62, 1017-1025. doi: 10.1037/0022-006X.62.5.1017

Choi, Y. Y., Shamosh, N. A., Cho, S. H., DeYoung, C. G., Lee, M. J., Lee, J. M., et al. (2008). Multiple bases of human intelligence revealed by cortical thickness and neural activation. J. Neurosci. 28, 10323-10329. doi: 10.1523/JNEUROSCI.325908.2008

Conway, A. R. A., Kane, M. J., and Engle, R. W. (2003). Working memory capacity and its relation to general intelligence. Trends Cogn. Sci. 7, 547-552. doi: 10.1016/j.tics.2003.10.005

Dale, A. M., Fischl, B., and Sereno, M. I. (1999). Cortical surface-based analysis. I. Segmentation and surface reconstruction. Neuroimage 9, 179-194. doi: 10.1006/nimg.1998.0395

Eden, G. F., and Zeffiro, T. A. (1998). Neural systems affected in developmental dyslexia revealed by functional neuroimaging. Neuron 21, 279-282. doi: 10.1016/S0896-6273(00)80537-1

Fletcher, J. M., Lyon, G. R., Fuchs, L., and Barnes, M. (2007). Learning Disabilities: From Identification to Intervention. New York: Guilford Press.

Fletcher, J. M., Shaywitz, S. E., Shankweiler, D. P., Katz, L., Liberman, I. Y., Stuebing, K. K., et al. (1994). Cognitive profiles of reading disability: comparisons of discrepancy and low achievement definitions. J. Educ. Psychol. 86, 6-23. doi: 10.1037/0022-0663.86.1.6

Francis, D. J., Shaywitz, S. E., Stuebing, K. K., Shaywitz, B. A., and Fletcher, J. M. (1996). Developmental lag versus deficit models of reading disability: a longitudinal, individual growth curve analysis. J. Educ. Psychol. 88, 3-17. doi: 10.1037/0022-0663.88.1.3

Gläscher, J., Rudrauf, D., Colom, R., Paul, L. K., Tranel, D., Damasio, H., et al. (2010). Distributed neural systems for general intelligence revealed by lesion mapping. Proc. Natl. Acad. Sci. U.S.A. 107, 4705-4709. doi: 10.1073/pnas.0910397107

Hämäläinen, M. S., and Ilmoniemi, R. J. (1994). Interpreting magnetic fields of the brain: minimum norm estimates. Med. Biol. Eng. Comput. 32, 35-42. doi: 10.1007/BF02512476

Hayes, A. F. (2013). Model Templates for Process for SPSS and SAS [WWW Document]. Available at: http://afhayes.com/introduction-to-mediation-moderationand-conditional-process-analysis.html. (accessed May 10, 2013).

Hoeft, F., Meyler, A., Hernandez, A., Juel, C., Taylor-Hill, H., Martindale, J. L., et al. (2007a). Functional and morphometric brain dissociation between dyslexia and reading ability. Proc. Natl. Acad. Sci. U.S.A. 104, 4234-4239. doi: 10.1073/pnas.0609399104

Hoeft, F., Ueno, T., Reiss, A. L., Meyler, A., Whitfield-Gabrieli, S., Glover, G. H., et al. (2007b). Prediction of children's reading skills using behavioral, functional, and structural neuroimaging measures. Behav. Neurosci. 121, 602-613. doi: 10.1037/0735-7044.121.3.602

Hoskyn, M., and Swanson, H. L. (2000). Cognitive processing of low achievers and children with reading disabilities: a selective meta-analytic review of the published literature. Sch. Psychol. Rev. 29, 102-119.

Jacquemot, C., Pallier, C., LeBihan, D., Dehaene, S., and Dupoux, E. (2003). Phonological grammar shapes the auditory cortex: a functional magnetic resonance imaging study. J. Neurosci. 23, 9541-9546.
Jobard, G., Crivello, F., and Tzourio-Mazoyer, N. (2003). Evaluation of the dual route theory of reading: a metanalysis of 35 neuroimaging studies. Neuroimage 20, 693-712. doi: 10.1016/S1053-8119(03)00343-4

Joseph, J., Noble, K., and Eden, G. (2001). The neurobiological basis of reading. J. Learn. Disabil. 34, 566-579. doi: 10.1177/002221940103400609

Kamara, S., Ad-Dab’bagh, Y., Haier, R. J., Deary, I. J., Lyttelton, O. C., Lepage, C., et al. (2009). Positive association between cognitive ability and cortical thickness in a representative US sample of healthy 6 to 18 year-olds. Intelligence 37, 145-155. doi: 10.1016/j.intell.2008.09.006

Kroger, J. K., Sabb, F. W., Fales, C. L., Bookheimer, S. Y., Cohen, M. S., and Holyoak, K. J. (2002). Recruitment of anterior dorsolateral prefrontal cortex in human reasoning: a parametric study of relational complexity. Cereb. Cortex 12, 477-485. doi: $10.1093 /$ cercor/12.5.477

Maisog, J. M., Einbinder, E. R., Flowers, D. L., Turkeltaub, P. E., and Eden, G. F. (2008). A Meta-analysis of functional neuroimaging studies of dyslexia. Ann. N. Y. Acad. Sci. 1145, 237-259. doi: 10.1196/annals.1416.024

Majerus, S., Linden, M. V., Collette, F., Laureys, S., Poncelet, M., Degueldre, C., et al. (2005). Modulation of brain activity during phonological familiarization. Brain Lang. 92, 320-331. doi: 10.1016/j.bandl.2004.07.003

McCandliss, B. D., Cohen, L., and Dehaene, S. (2003). The visual word form area: expertise for reading in the fusiform gyrus. Trends Cogn. Sci. 7, 293-299. doi: 10.1016/S1364-6613(03)00134-7

McCrory, E. J., Mechelli, A., Frith, U., and Price, C. J. (2005). More than words: a common neural basis for reading and naming deficits in developmental dyslexia? Brain 128, 261-267. doi: 10.1093/brain/awh340

Morris, R. D., Stuebing, K. K., Fletcher, J. M., Shaywitz, S. E., Lyon, G. R., Shankweiler, D. P., et al. (1998). Subtypes of reading disability: variability around a phonological core. J. Educ. Psychol. 90, 347-373.

Osipowicz, K., Rickards, T., Shah, A., Sharan, A., Sperling, M., Kahn, W., et al. (2011). A test of the role of the medial temporal lobe in single-word decoding. Neuroimage 54, 1455-1464. doi: 10.1016/j.neuroimage.2010.09.049

Perfetti, B., Saggino, A., Ferretti, A., Caulo, M., Romani, G. L., Onofrj, M., et al. (2009). Differential patterns of cortical activation as a function of fluid reasoning complexity. Hum. Brain Mapp. 30, 497-510. doi: 10.1002/hbm.20519

Philipose, L. E., Gottesman, R. F., Newhart, M., Kleinman, J. T., Herskovits, E. H., Pawlak, M. A., et al. (2007). Neural regions essential for reading and spelling of words and pseudowords. Ann. Neurol. 62, 481-492. doi: 10.1002/ana.21182

Pugh, K. R., Mencl, W. E., Shaywitz, B. A., Shaywitz, S. E., Fulbright, R. K., Constable, R. T., et al. (2000). The angular gyrus in developmental dyslexia: task-specific differences in functional connectivity within posterior cortex. Psychol. Sci. 11, 51-56. doi: 10.1111/1467-9280.00214

Richards, T. L., Berninger, V. W., Aylward, E. H., Richards, A. L., Thomson, J. B., Nagy, W. E., et al. (2002). Reproducibility of proton MR spectroscopic imaging (PEPSI): comparison of dyslexic and normal-reading children and effects of treatment on brain lactate levels during language tasks. Am. J. Neuroradiol. 23, 16781685.

Roux, F. E., Lubrano, V., Lauwers-Cances, V., Trémoulet, M., Mascott, C. R., and Démonet, J. F. (2004). Intra-operative mapping of cortical areas involved in reading in mono- and bilingual patients. Brain 127, 1796-1810. doi: 10.1093/brain/awh204

Rutter, M., and Yule, W. (1975). The concept of specific reading retardation. J. Child Psychol. Psychiatry 16, 181-197. doi: 10.1111/j.1469-7610.1975.tb01269.x

Scott, S. K., Blank, C. C., Rosen, S., and Wise, R. J. (2000). Identification of a pathway for intelligible speech in the left temporal lobe. Brain 123, 2400-2406. doi: 10.1093/brain/123.12.2400

Shaw, P., Greenstein, D., Lerch, J., Clasen, L., Lenroot, R., Gogtay, N., et al. (2006). Intellectual ability and cortical development in children and adolescents. Nature 440, 676-679. doi: 10.1038/nature04513

Shaywitz, B. A., Shaywitz, S. E., Pugh, K. R., Mencl, W. E., Fulbright, R. K., Constable, R. T., et al. (2002). Disruption of posterior brain systems for reading in children with developmental dyslexia. Biol. Psychiatry 52, 101-110. doi: 10.1016/S00063223(02)01365-3

Shaywitz, S. E., Shaywitz, B. A., Fulbright, R. K., Skudlarski, P., Mencl, W. E., Constable, R. T., et al. (2003). Neural systems for compensation and persistence: Young adult outcome of childhood reading disability. Biol. Psychiatry 54, 25-33. doi: 10.1016/S0006-3223(02)01836-X

Shaywitz, S. E., Shaywitz, B. A., Pugh, K. R., Fulbright, R. K., Constable, R. T., Mencl, W. E., et al. (1998). Functional disruption in the organization of the 
brain for reading in dyslexia. Proc. Natl. Acad. Sci. U.S.A. 95, 2636-2641. doi: 10.1073/pnas.95.5.2636

Simos, P. G., Fletcher, J. M., Sarkari, S., Billingsley, R. L., Denton, C. A., and Papanicolaou, A. C. (2007). Altering the brain circuits for reading through intervention: a magnetic source imaging study. Neuropsychology 21, 485-496. doi: 10.1037/0894-4105.21.4.485

Simos, P. G., Rezaie, R., Fletcher, J. M., Juranek, J., Passaro, A. D., Li, Z., etal. (2011). Functional disruption of the brain mechanism for reading: effects of comorbidity and task difficulty among children with developmental learning problems. Neuropsychology 24, 520-534. doi: 10.1037/ a0022550

Simpson, G. V., Weber, D. L., Dale, C. L., Pantazis, D., Bressler, S. L., Leahy, R. M., et al. (2011). Dynamic activation of frontal, parietal, and sensory regions underlying anticipatory visual spatial attention. J. Neurosci. 31, 13880-13889. doi: 10.1523/JNEUROSCI.1519-10.2011

Specht, K., Holtel, C., Zahn, R., Herzog, H., Krause, B. J., Motthagy, F. M., et al. (2003). Lexical decision of nonwords and pseudowords in humans: a positron emission tomography study. Neurosci. Lett. 345, 177-181. doi: 10.1016/S03043940(03)00494-4

Stanovich, K. E., and Siegel, L. S. (1994). Phenotypic performance profile of children with reading disabilities: a regression-based test of the phonological-core variable-difference model. J. Educ. Psychol. 86, 24-53. doi: 10.1037/0022-0663. 86.1.24

Stuebing, K. K., Barth, A. E., Molfese, P. J., Weiss, B., and Fletcher, J. M. (2009). IQ is not strongly related to response to reading instruction: a meta-analytic interpretation. Except. Child. 76, 31-51.

Stuebing, K. K., Fletcher, J. M., LeDoux, J. M., Lyon, G. R., Shaywitz, S. E., and Shaywitz, B. A. (2002). Validity of IQ-discrepancy classifications of reading disabilities: a meta-analysis. Am. Educ. Res. J. 39, 469-518. doi: 10.3102/000283120390 02469

Swanson, J. (1992). School-Based Assessments and Interventions for ADD Children. Irvine, CA: K.C. Publications.

Taki, Y., Hashizume, H., Sassa, Y., Takeuchi, H., Asano, M., Asano, K., et al. (2012). Correlation among body height, intelligence, and brain gray matter volume in healthy children. Neuroimage 59, 1023-1027. doi: 10.1016/j.neuroimage.2011.08.092

Tamnes, C. K., Ostby, Y., Walhovd, K. B., Westlye, L. T., Due-Tonnessen, P., and Fjell, A. M. (2010). Intellectual abilities and white matter microstructure in development: a diffusion tensor imaging study. Hum. Brain Mapp. 31, 1609-1625. doi: 10.1002/hbm.20962

Tanaka, H., Black, J. M., Hulme, C., Stanley, L. M., Kesler, S. R., WhitfieldGabrieli, S., et al. (2011). The brain basis of the phonological deficit in dyslexia is independent of IQ. Psychol. Sci. 22, 1442-1451. doi: 10.1177/09567976114 19521

Tarkiainen, A., Helenius, P., Hansen, P. C., Cornelissen, P. L., and Salmelin, R. (1999). Dynamics of letter string perception in the human occipito-temporal cortex. Brain 122, 2119-2132. doi: 10.1093/brain/122.11.2119
Temple, E., Poldrack, R. A., Salidis, J., Deutsch, G. K., Tallal, P., Merzenich, M. M., et al. (2001). Disrupted neural responses to phonological and orthographic processing in dyslexic children: an fMRI study. Neuroreport 12, 299-307. doi: 10.1097/00001756-200102120-00024

van der Mark, S., Bucher, K., Mauer, U., Schulz, E., Brem, S., Buckelmuller, J., et al. (2009). Children with dyslexia lack multiple specializations along the visual word form (VWF) system. Neuroimage 47, 1940-1949. doi: 10.1016/j.neuroimage.2009.05.021

Vanni, S., Tanskanen, T., Seppa, M., Uutela, K., and Hari, R. (2001). Coinciding early activation of the human primary visual cortex and anteromedial cuneus. Proc. Natl. Acad. Sci. U.S.A. 98, 2776-2780. doi: 10.1073/pnas.041600898

Vellutino, F. R., Scanlon, D. M., and Lyon, G. R. (2000). Differentiating between difficult-to-remediate and readily remediated poor readers: more evidence against the IQ-achievement discrepancy definition of reading disability. J. Learn. Disabil. 33, 223-238. doi: 10.1177/002221940003300302

Wadsworth, S. J., Olson, R. K., and DeFries, J. C. (2010). Differential genetic etiology of reading difficulties as a function of IQ: an update. Behav. Genet. 40, 751-758. doi: 10.1007/s10519-010-9349-x

Wechsler, D. (1999). Wechsler Abbreviated Scale of Intelligence (WASI). San Antonio, TX: The Psychological Corporation.

Wiley, J., Jarosz, A. F., Cushen, P. J., and Colflesh, G. J. H. (2011). New rule use drives the relation between working memory capacity and Raven's advanced progressive matrices. J. Exp. Psychol. Learn. Mem. Cogn. 37, 256-263. doi: 10.1037/a0021613

Woodcock, R. W., McGrew, K. S., and Mather, N. (2001). Woodcock-Johnson III Tests of Achievement. Itasca, IL: Riverside.

Wu, K., Taki, Y., Sato, K., Hashizume, H., Sassa, Y., Takeuchi, H., et al. (2013). Topological organization of functional brain networks in healthy children: differences in relation to age, sex, and intelligence. PLOS ONE 8:e55347. doi: 10.1371/journal.pone.0055347

Conflict of Interest Statement: The authors declare that the research was conducted in the absence of any commercial or financial relationships that could be construed as a potential conflict of interest.

Received: 03 August 2013; paper pending published: 21 October 2013; accepted: 23 December 2013; published online: 08 January 2014.

Citation: Simos PG, Rezaie R, Papanicolaou AC and Fletcher JM (2014) Does IQ affect the functional brain network involved in pseudoword reading in students with reading disability? A magnetoencephalography study. Front. Hum. Neurosci. 7:932. doi: 10.3389/fnhum.2013.00932

This article was submitted to the journal Frontiers in Human Neuroscience. Copyright (c) 2014 Simos, Rezaie, Papanicolaou and Fletcher. This is an open-access article distributed under the terms of the Creative Commons Attribution License (CC BY). The use, distribution or reproduction in other forums is permitted, provided the original author(s) or licensor are credited and that the original publication in this journal is cited, in accordance with accepted academic practice. No use, distribution or reproduction is permitted which does not comply with these terms. 